\title{
Survie du phytoplasme de la flavescence dorée de la vigne en présence d'extraits d'hôtes insectes et végétaux
}

\author{
C Kuszala \\ Unité de recherches sur les phytoplasmes, Inra, BV 1540, F-21034 Dijon, France
}

(Reçu le 25 mars 1996 ; accepté le 27 septembre 1996)

\begin{abstract}
Summary - Inhibition of the pathogenicity of the grapevine Flavescence doree phytoplasma by host insect and plant extracts. We have tested the effect of host plant extracts (Catharanthus roseus, Vicia faba and Vitis vinifera) and host insect extracts (Euscelidius variegatus) on the pathogenicity of the Flavescence dorée (FD) phytoplasma. This pathogenicity was assessed by microinjection into healthy leafhoppers, followed by FD transmission onto healthy $\vee$ faba. We first present results on phytoplasma extraction. Data from infectious leafhopper extracts suggest that males were a better source of extraction than females. The extraction of phytoplasma from $V$ faba showed an inhibitory effect. Then, in order to study the inhibitory effect of plant and vector extracts on phytoplasma pathogenicity, we incorporated healthy extracts of these hosts into a preservation medium. We detected various degrees of toxicity according to the amount of extract with both plants and insects. In addition, insects showed differences in relation to their stages of development and sex. C roseus, a tropical plant, was the least toxic of all healthy host plants. It is interesting to note that this plant is known to be an experimental host for numerous cases of yellowing with extractible or non-extractible phytoplasma.
\end{abstract}

\section{host extract / phytoplasma / Flavescence dorée / pathogenicity / survival}

Résumé - Les effets d'interférence entraînés par la présence d'extraits d'insecte vecteur (Euscelidius variegatus) ou de végétaux hôtes (Vitis vinifera, Catharanthus roseus, Vicia faba) sur l'extraction et la survie du phytoplasme de la flavescence dorée (FD) de la vigne sont étudiés. L'épreuve d'infectivité utilisée est l'injection à des cicadelles saines. Dans un premier temps, nous présentons des résultats d'extraction des phytoplasmes. À partir d'insectes infectieux, les données suggèrent que les mâles sont une meilleure source d'extraction que les femelles. À partir de Vicia faba, un effet toxique sur les phytoplasmes lors de leur extraction est constaté. Dans un second temps, afin d'explorer cet effet toxique des extraits des insectes vecteurs et végétaux sur la viabilité des phytoplasmes, des extraits sains de ces hôtes ont été incorporés dans un milieu de survie. Suivant leur concentration, nous avons détecté une variation des effets toxiques de ces extraits sur les phytoplasmes. De plus, avec les insectes, nous avons constaté des différences de toxicité selon le stade et selon le sexe. Parmi les hôtes végétaux sains, le Catharanthus roseus, plante de climat tropical, est le moins toxique. Il est intéressant de noter que cette plante est connue pour être un hôte de laboratoire pour de nombreuses jaunisses à phytoplasmes extractibles ou non. 


\section{INTRODUCTION}

Ces dernières années, la connaissance des phytoplasmes, autrefois appelés MLO (Mycoplasmalike organisms), a considérablement progressé grâce aux techniques sérologiques et moléculaires. Ces études (Cousin, 1995) ont joué un rôle déterminant en permettant leur taxonomie (Seemüller et al, 1994). Cependant, ces techniques sérologiques et moléculaires ne peuvent remplacer ni l'extraction, ni l'isolement des phytoplasmes. En effet, par reproduction expérimentale de la maladie, cette extraction permet la mise en évidence de leur pouvoir pathogène (Caudwell et Kuszala, 1986).

Les phytoplasmes sont des microorganismes unicellulaires et pléiomorphes, de 80 à $1000 \mathrm{~nm}$ de diamètre, sans paroi, parasites obligatoires et à habitat intracellulaire. Ils se multiplient alternativement dans les cellules du phloème de la plante et dans celles de l'insecte vecteur (Moreau, 1990).

Au cours de l'extraction des agents pathogènes vivants, à partir de leurs hôtes insecte ou végétal, tout facteur agissant sur leur survie peut avoir son importance. Dans la littérature, Hamilton (1934) puis Black (1939) signalent déjà un effet toxique de l'extrait d'insectes sur des virus. En 1986, Alivizatos et Markham ont démontré un effet inhibiteur de l'extrait d'insecte vecteur sur la multiplication du spiroplasme du Corn Stunt. En étudiant l'effet d'extrait de plantes sur des mycoplasmes animaux et sur Acholeplasma laidlawii, Teranaka et al (1976), ont mis en évidence un effet inhibiteur du Paulownia plus important sur Mycoplasma bovine que sur Acholeplasma laidlawii. Bien que non pathogène pour les plantes, Acholeplasma a été isolé à partir de végétaux (Eden-Green, 1978), et des études sur sa multiplication in vitro en présence d'une infusion de plante confirment sa faible sensibilité à cette présence (Ivanov et al, 1992). Cet effet toxique de l'extrait végétal a également été étudié sur des bactéries (Majidi et al, 1992). À ce jour, l'incidence des extraits d'hôtes sur le pouvoir pathogène des phytoplasmes a été suggérée mais jamais démontrée. Notre présente contribution a été de mettre en évidence cet effet.

La maladie à phytoplasmes étudiée ici est la flavescence dorée (FD) de la vigne. Les étapes qui ont permis d'adapter ce modèle au travail de laboratoire ont été la transmission à la fève Vicia faba L (Caudwell et al, 1970), I'utilisation d'une cicadelle indigène, Euscelidius variegatus KBM, pour la transmission de la maladie d'une fève à une autre (Caudwell et al, 1972) puis la mise au point d'une épreuve d'infectivité sensible et fidèle (Caudwell, 1977 ; Kuszala, 1986). L'extraction du phytoplasme de la FD a été obtenue à partir de Catharanthus roseus (Caudwell et al, 1973), à partir de cicadelles (Caudwell et Kuszala, 1986) et à partir de $V$ faba (Caudwell et al, 1988). Chez les cicadelles, les phytoplasmes se trouvent en grande partie dans les glandes salivaires et les intestins (Caudwell et al, 1971; Lherminier et al, 1990 ; Lefol et al, 1994). Chez les plantes, les phytoplasmes se rencontrent principalement dans les tubes criblés jeunes et adultes (Caudwell et al, 1971 ; Granata et Grimaldi, 1991 ; Meignoz et al, 1992). Actuellement les domaines les plus obscurs concernent les bases moléculaires du parasitisme qui gouvernent leur transmission par les cicadelles, et leur pathogénicité qui induit chez la plante des désordres caractéristiques. Cette ignorance tient essentiellement au fait qu'à ce jour aucun phytoplasme n'est cultivable ex vivo (Sears et Schewe, 1994) et qu'ils sont très difficilement séparables par des méthodes biochimiques des composants cellulaires de l'hôte infecté.

L'objectif de ce travail a été d'étudier la persistance du pouvoir pathogène du phytoplasme de la FD de la vigne en présence d'extraits d'hôtes animaux et végétaux. Cette pathogénicité a été évaluée par micro-injection au vecteur sain suivie d'une reproduction expérimentale de la maladie. Nous avons, dans un premier temps, interprété les résultats d'extraction des phytoplasmes en fonction de leurs origines. Nous nous sommes ensuite intéressée à l'influence in vitro des extraits d'insectes et de végétaux sur la survie du phytoplasme de la FD.

\section{MATÉRIEL ET MÉTHODES}

\section{Milieux}

Le milieu d'extraction utilisé est le 1379. Sa composition (Caudwell et Kuszala, 1986) est la suivante : NCTC $135: 20 \%$, Grace modifié : $42 \%$, fotal bovine sérum chauffé 30 minutes à $56{ }^{\circ} \mathrm{C}: 13 \%$, eau ultrapure : $25 \%$. Le pH a été ajusté à 7 avec une solution de $\mathrm{KOH}$. Pour les extraits d'insectes sains, le milieu a été complété avec $0,5 \%$ de polyvinylpyrrolidone $\mathrm{K}-30$ (PVP). Pour les extraits de végétaux sains, on a ajouté les produits suivants : glutathion $0,2 \mathrm{mg} / \mathrm{mL}$, histidine $\mathrm{HCl}$ monohydrate $4,8 \mathrm{mg} / \mathrm{mL}$, histidine base $4,8 \mathrm{mg} / \mathrm{mL}$ (Caudwell et al, 1988). Pour les fèves malades, on a ajouté $2 \%$ de PVP au mélange précédant. 
L'effet du pH sur la survie (18 heures à $23^{\circ} \mathrm{C}$ ) des phytoplasmes a été étudié pour différentes valeurs du $\mathrm{pH}$ du milieu $1379(\mathrm{pH}$ ajusté avec une solution de $\mathrm{KOH}): 6,6 ; 7 ; 7,8$ et 8,2 .

\section{Extraction et infectivité du phytoplasme de différentes origines}

\section{Extraits d'insectes infectieux}

Nous avons réalisé toutes nos expériences avec la cicadelle $E$ variegatus (Caudwell et Larrue, 1977). Nous avons utilisé des insectes infectieux, d'une part pour étudier le rendement en phytoplasmes au cours de leur extraction et d'autre part pour faire des filtrats infectants servant à tester les conditions de $\mathrm{pH}$ et l'effet des extraits sains (insectes et plantes) sur la survie des phytoplasmes.

Pour déterminer le meilleur rendement en phytoplasmes, nous avons (dans quatre essais différents : essais I à IV) mené en parallèle des extractions de phytoplasmes à partir de cicadelles mâles et femelles infectieuses (avec six insectes par $\mathrm{mL}$ de milieu 1379). Pour chaque extrait de phytoplasmes, nous avons effectué une dilution au $1 / 50^{\circ}$ dans le même milieu suivi d'une congélation à $-70^{\circ} \mathrm{C}$.

II nous a paru intéressant d'étudier quatre groupes de milieux susceptibles d'affecter la survie (18 heures) des phytoplasmes de la FD : pH du milieu 1379, extraits sains d'insectes, extraits sains de plantes, extraits sains d'insectes en fonction du $\mathrm{pH}$. Pour chaque groupe étudié, nous avons préparé un filtrat infectant avec six mâles infectieux par $\mathrm{mL}$ de milieu 1379. Le filtrat infectant a été incorporé au $1 / 50^{\mathrm{e}}$ dans les milieux composant le groupe à étudier.

\section{Extraits de fèves malades}

Nous avons extrait en parallèle les phytoplasmes séparément du sommet de la tige et des feuilles correspondant au même niveau à raison de 2,5\% (poids/volume) de matériel végétal. Les filtrats infectants ont été dilués au 1/2 dans du milieu 1379 et congelés à $-70^{\circ} \mathrm{C}$.

Pour détecter un effet toxique sur les phytoplasmes au cours de leur changement de milieu, nous avons fait trois extraits infectants à partir de sommets de tige de fèves présentant les symptômes de la maladie. Deux extractions de phytoplasmes ont été faites avec $12 \%$ (poids/volume) de matériel végétal (essais $V$ et VI) et une avec $25 \%$ (essai VII). Le filtrat infectant de l'essai $V$ a été divisé en deux, une fraction a été mise à $-70^{\circ} \mathrm{C}$ immédiatement et l'autre a été gardée 1 heure à $+4^{\circ} \mathrm{C}$. Une fraction des filtrats infectants des essais VI et VII a été diluée (dans du milieu 1379) au $1 / 5^{\mathrm{e}}$ pour l'essai $\mathrm{VI}$ et au $1 / 10^{\mathrm{e}}$ pour l'essai $\mathrm{VII}$, puis les liquides infectants dilués et non dilués ont été gardés 1 heure à $+4{ }^{\circ} \mathrm{C}$, puis congelés à $-70^{\circ} \mathrm{C}$.

\section{Action des extraits sains ajoutés}

\section{Extraits d'insectes sains}

Pour étudier l'effet des extraits d'insectes sains sur la viabilité des phytoplasmes de la FD, nous avons utilisé des mâles, des femelles et des larves. Nous utilisons couramment comme unité de mesure un nombre d'insectes à broyer par $\mathrm{mL}$. Dans ce protocole, nous avons voulu tenir compte de la correspondance entre le nombre d'insectes et le poids par $\mathrm{mL}$. Comme le poids des femelles est sujet à fluctuations selon la présence d'œufs, nous avons sélectionné un lot de six femelles pesant le poids (33 mg) d'un lot de douze mâles. Pour les larves, nous avons prélevé des larves du $1^{\mathrm{er}}$ au $5^{\mathrm{e}}$ stade jusqu'à obtention de $33 \mathrm{mg}$. Trois types d'extraits d'insectes sains de $33 \mathrm{mg} / \mathrm{mL}$ chacun ont donc été préparés : des larves du $1^{\mathrm{er}}$ au $5^{\mathrm{e}}$ stade, des mâles soit $12 / \mathrm{mL}$, des femelles soit $6 / \mathrm{mL}$. Pour chacun des trois types d'extraits, les dilutions utilisées ont été 1 et $1 / 2$. Ces différents extraits sains ont reçu une quantité uniforme $\left(1 / 50^{e}\right)$ d'un même filtrat infectant (tableau 1). Pour la majorité des liquides infectants, nous avons prélevé des aliquotes que nous avons immédiatement mises à congeler à $-70^{\circ} \mathrm{C}$ (temps : 0 ). Les liquides infectants ont été mis 18 heures à $23^{\circ} \mathrm{C}$ puis congelés. L'essai de survie, en présence de six femelles saines par $\mathrm{mL}$ a été noté $A$, et celui en présence de six mâles sains par $\mathrm{mL}$ obtenu par dilution $1 / 2$ de l'extrait à douze mâles par $\mathrm{mL}$, a été noté $\mathrm{B}$.

Pour étudier l'effet du pH sur la survie (18 heures à $23^{\circ} \mathrm{C}$ ) des phytoplasmes en présence d'extrait de larves saines, nous avons utilisé l'extrait sain de larves à $33 \mathrm{mg}$ par $\mathrm{mL}$, nous l'avons ensuite dilué au $1 / 2$ dans du milieu 1379 , puis ajusté aux $\mathrm{pH}: 6,6 ; 7 ; 7,4$ et 7,8 . Ensuite nous avons ajouté une quantité uniforme d'un même filtrat infectant (dilution $1 / 50^{\mathrm{e}}$ ). Les liquides infectants ont été gardés 18 heures à $23^{\circ} \mathrm{C}$, puis congelés à $-70^{\circ} \mathrm{C}$. .

\section{Extraits de plantes saines}

Nous avons travaillé avec la fève $V$ faba $L$, la pervenche de Madagascar $C$ roseus $\mathrm{L}$ et la vigne Vitis vinifera (variété Baco 22A). Les expériences d'extraction à partir de plantes saines ont été limitées à des sommets de tige. Ce choix s'appuie sur des travaux antérieurs concernant l'extraction des phytoplasmes à partir de pervenche (Caudwell et al, 1973) et de fève (Caudwell et al, 1988). Pour chaque espèce, quatre sommets de tige de $0,1 \mathrm{~g}$ chacun ont été utilisés pour $16 \mathrm{~mL}$ de milieu. Les extraits sains ont été utilisés aux dilutions $1,1 / 10$ et $1 / 100$ dans le milieu 1379 à pH 7 contenant du glutathion et les histidines (L-histidine et L-histidine monohydrochloride). Un témoin milieu, sans extrait de plante, a été inclu dans cette étude. Chaque liquide a reçu une quantité uniforme d'un même filtrat infectant $\left(1 / 50^{\mathrm{e}}\right)$. Les liquides infectants ont été gardés 18 heures à $+4{ }^{\circ} \mathrm{C}$ puis congelés à $-70^{\circ} \mathrm{C}$. 
Tableau I. Méthode de comparaison de différents extraits d'hôtes insectes sains avec un même filtrat de phytoplasmes.

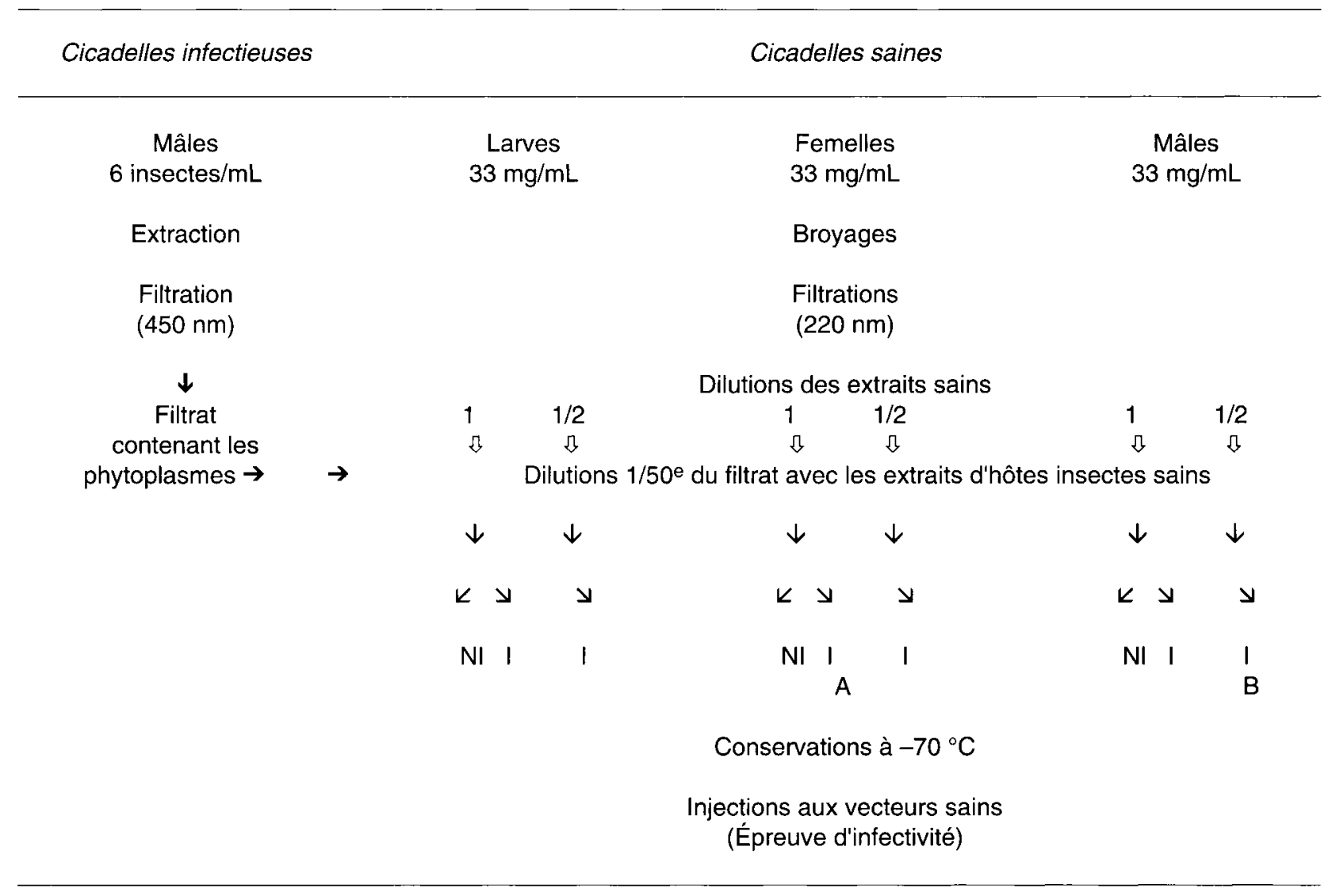

$\mathrm{I}$ : liquide infectant incubé 18 heures à $23^{\circ} \mathrm{C}$. $\mathrm{NI}$ : liquide infectant non incubé.

\section{Méthodes de broyage et clarifications}

Pour les plantes, on a effectué un découpage très fin des tissus avec des lames de rasoir ajustées sur les porte-lames d'un couteau électrique. Puis, on a complété le broyage à l'aide d'un Potter à main. Pour les plantes saines, le broyat a été clarifié à $2900 \mathrm{~g}$ pendant 30 minutes à $+4{ }^{\circ} \mathrm{C}$ dans une centrifugeuse horizontale Sigma $2 \mathrm{~K}$. Le surnageant a été préfiltré sur des filtres en profondeur du type Millipore AP 25, AP 20 et AP 15 montés en série. Le pH a été réajusté à 7.

Les insectes sains ont été broyés au Potter de Thomas, puis les broyats ont été clarifiés à $2900 \mathrm{~g}$. Les surnageants ont été centrifugés à $13300 \mathrm{~g}$ pendant 30 minutes à $+4^{\circ} \mathrm{C}$. Pour les insectes malades et les fèves malades après le broyage au Potter, les broyats ont été clarifiés dans une centrifugeuse horizontale à $2900 \mathrm{~g}, 5$ minutes à $+4{ }^{\circ} \mathrm{C}$. Puis les surnageants ont été préfiltrés sur deux disques en fibre de verre (Whatmann GFA, GFF), disposés en série sur un même support.

Les extraits de plantes saines et d'insectes infectieux ont été ensuite filtrés sur une membrane de porosité de $450 \mathrm{~nm}$ type HA Millipore, les extraits d'insectes sains sur $220 \mathrm{~nm}$.
Toutes les expériences d'extraction ont été menées en vitrine réfrigérée à $+4{ }^{\circ} \mathrm{C}$ et les manipulations des essais de survie des phytoplasmes ont été faites dans des conditions aseptiques (hotte à flux d'air laminaire, matériel stérile).

\section{Conservation des suspensions de phytoplasmes}

Au terme des expériences, toutes les suspensions de phytoplasmes (sans incubation ou après incubations) ont été congelées à $-70^{\circ} \mathrm{C}$ jusqu'à l'épreuve d'infectivité. Cette congélation permet d'étaler dans le temps les épreuves d'infectivité et évite ainsi d'en laisser certaines en attente.

\section{Épreuve d'infectivité}

Le but de l'épreuve d'infectivité est d'apporter une évaluation de la concentration d'une suspension en unités (ou particules) infectieuses. À ce jour, l'infectivité d'une suspension de phytoplasmes ne peut être évaluée que 
par l'injection de la suspension dans le corps d'insectes vecteurs sains suivie de la détermination de leur infectivité. Ainsi, l'épreuve d'infectivité de la FD est réalisée en injectant la cicadelle $E$ variegatus puis en utilisant la plante $V$ faba comme révélateur de l'infectivité des cicadelles injectées. Nous concevons donc ainsi l'épreuve d'infectivité.

\section{Technique d'inoculation}

Les insectes sont anesthésiés par le gaz carbonique sous un éclairage froid à fibres optiques. Leur inoculation est réalisée avec une pipette Pasteur étirée en capillaire très fin. L'injection se fait dans le flanc de l'insecte entre le $4^{\mathrm{e}}$ et $5^{\mathrm{e}}$ segments, en biais, la pointe de la pipette dirigée vers la tête de l'insecte. La pipette Pasteur est reliée par un tube souple à la bouche de l'opérateur qui module ainsi la pression ; celle-ci est arrêtée au premier gonflement de l'insecte, qui s'accompagne d'un suintement de liquide au niveau de la piqûre. Nous injectons ainsi chaque suspension de phytoplasmes à 30 jeunes mâles sains (Kuszala, 1986).

\section{Manipulation des insectes après l'injection}

Les 30 insectes injectés avec une suspension de phytoplasmes (fig 1) sont placés sous cagette sur deux fèves ( $A$ et $B$ ) pendant les 21 jours nécessaires à l'incubation de l'infectivité dans le vecteur. Les insectes survivants au bout de ces 3 semaines sont répartis à raison de trois par fève, pendant 2 semaines, sur de jeunes fèves saines (servant de révélateur de l'infectivité des cicadelles et numérotées de 1 à 4 dans l'exemple de la figure 1). Le nombre de ces insectes survivants étant élevé, il n'a pas été possible de répartir les cicadelles injectées à raison d'une par plante (pour des raisons de place). En effet, ces plantes doivent séjourner deux semaines en insectarium pour leur

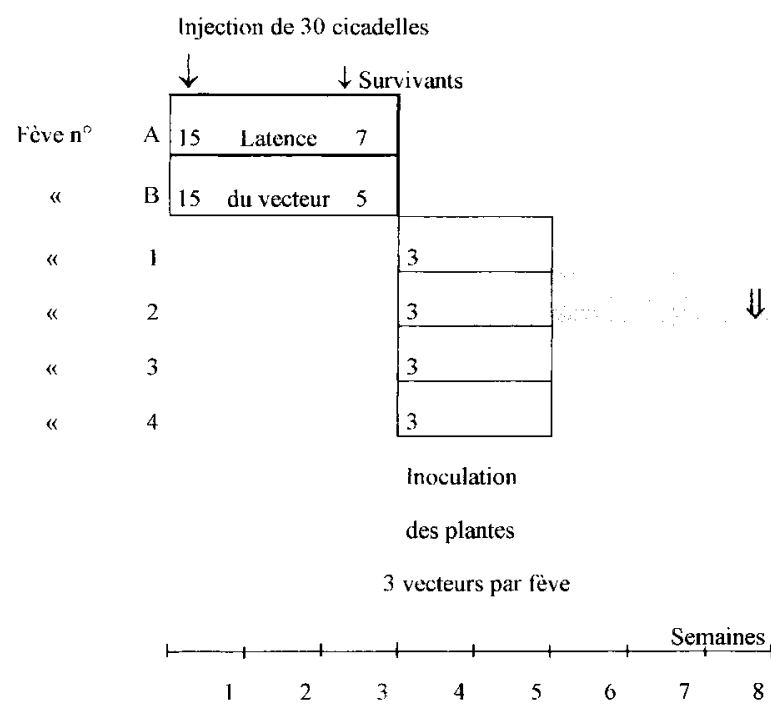

Fig 1. Épreuve d'infectivité d'une suspension de phytoplasmes. incubation de la maladie dans la plante $; \Omega$ : apparition des symptômes sur la plante. infection, puis trois semaines en moyenne en serre en attente des symptômes. Nous avons donc regroupé sur chaque fève, sous cagette, pendant deux semaines, trois cicadelles injectées.

\section{Plantes révélatrices de l'infectivité des cicadelles injectées}

Au bout des 2 semaines, les cicadelles sont tuées et les plantes sont placées en serre en conditions favorables à la manifestation des symptômes.

\section{Rappel de la méthode de traitement des résultats (Caudwell, 1977)}

Dans ces conditions, si les cicadelles sont groupées à raison de plusieurs par plante, la variable à prendre en considération n'est pas le nombre de cicadelles devenues infectieuses, mais le nombre de plantes devenues malades. Nous avons appliqué le calcul statistique qui permet de déduire le nombre de particules infectieuses par $\mathrm{mL}$ de solution du pourcentage de plantes malades (fig 2 : rappel de l'abaque).

Dans notre cas, nous considérerons comme effet toxique toute action diminuant le nombre d'unités infectieuses par $\mathrm{mL}(\mathrm{U} / / \mathrm{mL})$. Cet effet se traduit directement par une diminution du nombre de fèves qui expriment des symptômes par rapport au nombre total des fèves inoculées par les cicadelles injectées. Le nombre total des fèves inoculées par chaque suspension de phytoplasmes est conditionné par le nombre d'insectes survivants au bout des vingt et un jours d'incubation. Ce nombre est variable d'une épreuve d'infectivité à l'autre. En effet, dans un système aussi long et complexe (mettant en relation le vecteur, l'agent pathogène et la plante), de nombreux facteurs peuvent intervenir (prédateurs des cicadelles, parasites des plantes, etc). Pour les épreuves d'infectivité, nous nous sommes limitée à une seule dilution pour des raisons d'espace disponible. En effet, sauf pour les expériences faites à partir de fèves malades, toutes les suspensions de phytoplasmes injectées aux insectes sont des dilutions au $1 / 50^{e}$, valeur choisie qui dans nos conditions permet la mise en évidence de l'infectivité et l'exploitation de nos résultats.

\section{Exemple d'utilisation de l'abaque (fig 2)}

Le sens des opérations est donné par des flèches sur les lignes en trait interrompu.

Lorsque l'on a quatre fèves malades sur cinq, soit $80 \%$ de plantes malades, chacune ayant hébergé trois cicadelles inoculées $(n=3)$ on a $\mathrm{P}_{3}=0,2$.

\section{Détermination du nombre moyen de particules infectieuses par $m L$}

On suit la ligne horizontale $P_{3}=0,2$ jusqu'à son intersection $A$ avec la droite $n=3$. La projection de ce point A sur l'axe des abscisses donne la valeur de $M$ 


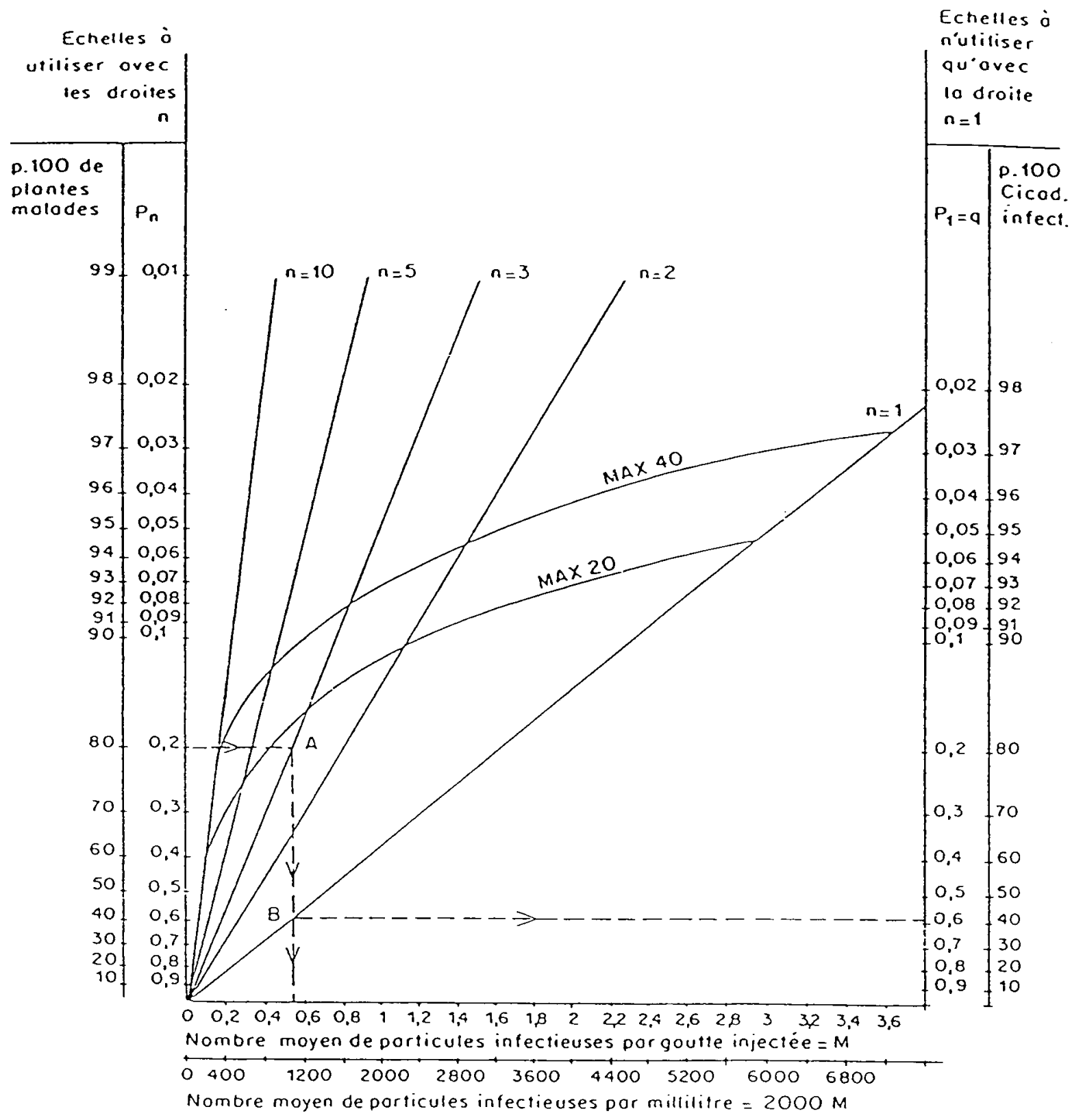

Fig 2. Rappel de l'abaque (Caudwell, 1977) mettant en relation les pourcentages observés de plantes malades et le nombre moyen de particules infectieuses par unité de volume de la solution injectée, selon le nombre $n$ de cicadelles par plante. $q$ : probabilité qu'une cicadelle ne soit pas infectée ; $P_{1}$ : probabilité pour qu'une plante ayant reçu une cicadelle ne soit pas infectée; $n:$ nombre de cicadelles placées sur une plante $; P_{n}$ : proportion de plantes non malades ayant reçu $n$ cicadelles par plante.

(nombre moyen de particules infectieuses par goutte injectée) et de $2000 \mathrm{M}$ (nombre moyen de particules infectieuses par $\mathrm{mL}$ de la solution injectée).

\section{Détermination des pourcentages de plantes malades que l'on aurait obtenus avec 1, 2, $5 \ldots$ $\mathrm{n}$ cicadelles par plante.}

La droite verticale passant par $A$ rencontre les droites $n=1, n=2, n=5$, etc, en des points dont les ordonnées sont égales aux proportions $P_{1}, P_{2}, P_{5}$, etc, de plantes non malades que l'on aurait obtenues en mettant $1,2,5$, etc cicadelles par plante.

\section{Détermination du pourcentage de cicadelles infectieuses}

L'intersection $B$ de cette droite verticale passant par A avec la droite $n=1$ est particulièrement intéressante car $P_{1}=q$ (probabilité qu'une cicadelle ne soit pas infectée). Le point B se trouve donc à l'ordonnée $q(\mathrm{On}$ peut le projeter sur l'axe des $q$ représenté en ordonnées sur la droite de l'abaque). Cet axe est doublé par un autre indiquant les pourcentages de cicadelles infectieuses.

Lorsque l'extrait ou la culture a été concentré ou dilué avant l'injection, on passe des valeurs de $M$ trou- 
vées pour la solution injectée aux valeurs réelles en multipliant $\mathrm{M}$ par le rapport de concentration.

\section{RÉSULTATS}

\section{Interprétation de l'épreuve d'infectivité d'une suspension de phytoplasmes (diluée au 1/50)}

Dans le cas de la figure 1, nous obtenons une fève malade sur quatre, soit $25 \%$ de plantes malades, valeur que nous reportons sur l'abaque de la figure 2. Nous en déduisons :

- au minimum $27 \%$ de cicadelles sont devenues infectieuses ;

- nous avons inoculé en moyenne 0,1 particule infectieuse par cicadelle.

Connaissant le volume de la goutte injectée (estimé à $0,0005 \mathrm{~mL}$ ), on peut en déduire (valeur lue 200) la valeur minimum $200 \times 50$ (car dilution au $1 / 50^{\mathrm{e}}$ ) $=10^{4}$ particules (ou unités) infectieuses par $\mathrm{mL}$.

\section{Extraits d'insectes}

\section{Extractions des phytoplasmes à partir d'E variegatus infectieux}

Dans la figure $3 a$, nous comparons les résultats d'extraction de phytoplasmes menées en parallèle à partir de six mâles et de six femelles par $\mathrm{mL}$ du milieu 1379 dans quatre essais (I à IV) effectués à des dates différentes. Les données suggèrent que les mâles sont une meilleure source d'extraction de phytoplasmes que les femelles.

\section{Effets des différents extraits d'E variegatus sains sur la viabilité des phytoplasmes}

La figure 4 donne les résultats de la survie des phytoplasmes de la FD au temps 0 et au bout de 18 heures à $23^{\circ} \mathrm{C}$ (méthode de comparaison : tableau I). Au temps 0 et à la dilution 1 (33 $\mathrm{mg}$ d'extrait sains par $\mathrm{mL}$ ), les résultats concernant les extraits mâles et femelles sains donnent $100 \%$ de plantes malades donc des résultats supérieurs à la limite de discrimination de l'épreuve. Nous ne pouvons pas détecter d'effet toxique. En revanche, en présence d'extrait de larves saines, une perte de viabilité des phytoplasmes est détectable dès le temps 0 . Au bout de 18 heures et à la dilution 1, en présence d'extraits de larves ou de femelles saines (essai A), nous constatons une perte de viabilité des phytoplasmes. Celle-ci est plus importante avec les femelles (essai $A$ ) où le résultat avec $0 \%$ de plante malade n'entre pas dans la limite de discrimination de l'épreuve. En revanche à la dilution $1 / 2$ de ces extraits sains, cette perte de viabilité n'est pas détectable. En présence d'extraits de mâles sains, une perte de viabilité est uniquement détectable à la dilution 1 íc au bout de 18 heures (essai $B$ ).

Dans la figure 5 les études de survie, 18 heures à $23^{\circ} \mathrm{C}$ et à différents $\mathrm{pH}$, indiquent un optimum à $\mathrm{pH} 7,4$ en présence d'extrait de larves saines (33

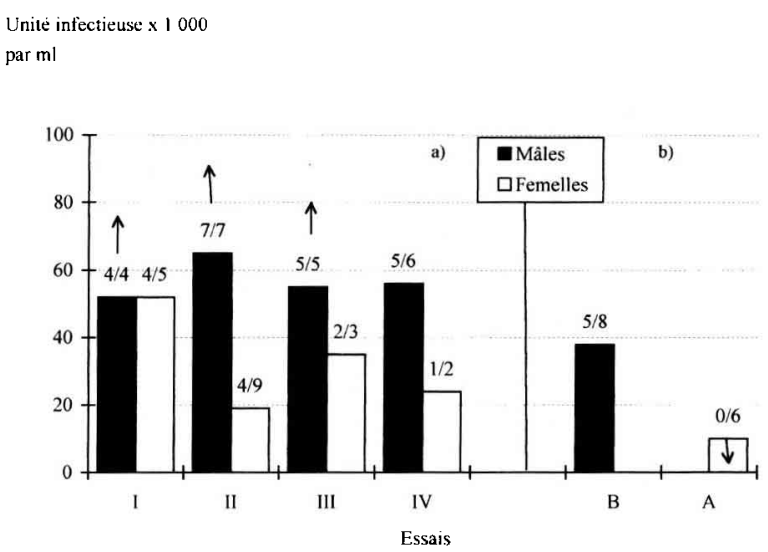

Fig 3. Comparaison de la viabilité du phytoplasme de la FD en présence d'extraits d' $E$ variegatus adultes (a) dans l'extrait de l'hôte infectieux (six insectes par $\mathrm{mL}$ ) dont il est issu, (b) en survie 18 heures à $23^{\circ} \mathrm{C}$ en présence d'extrait d'hôte sain : $A=$ six femelles par $\mathrm{mL} ; B=$ douze mâles par $\mathrm{mL}$ dilué $1 / 2$ dans du milieu 1379. ( $\uparrow$ : résultat supérieur et $\downarrow$ : résultat inférieur à la limite de discrimination de l'épreuve. Le rapport au-dessus de chaque barre correspond au nombre de fèves malades sur le nombre total de fèves inoculées par les cicadelles injectées).

Unite infectieuse $\times 1000$

par $\mathrm{ml}$

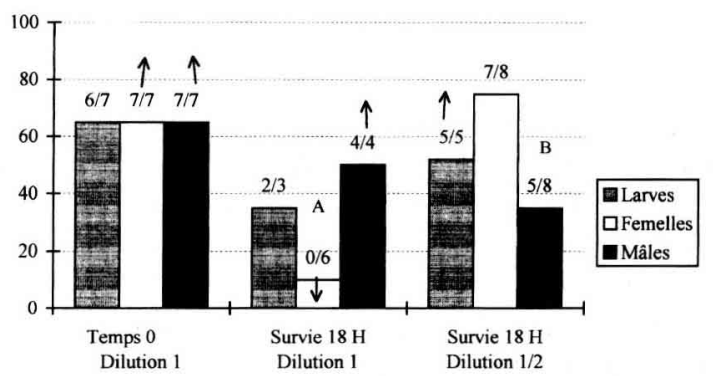

Fig 4. Étude de la toxicité des différents extraits d'E variegatus sains (larves, mâles et femelles) sur les phytoplasmes de la flavescence dorée de la vigne. $A=$ six femelles par $\mathrm{mL}$; $B=$ douze mâles par $m L$ dilué $1 / 2$ dans du milieu 1379. $(\uparrow$ : résultat supérieur et $\downarrow$ : résultat inférieur à la limite de discrimination de l'épreuve. Le rapport au-dessus de chaque barre correspond au nombre de fèves malades sur le nombre total de fèves inoculées par les cicadelles injectées). 
Unité infectieuse

$\times 1000$ par $\mathrm{ml}$

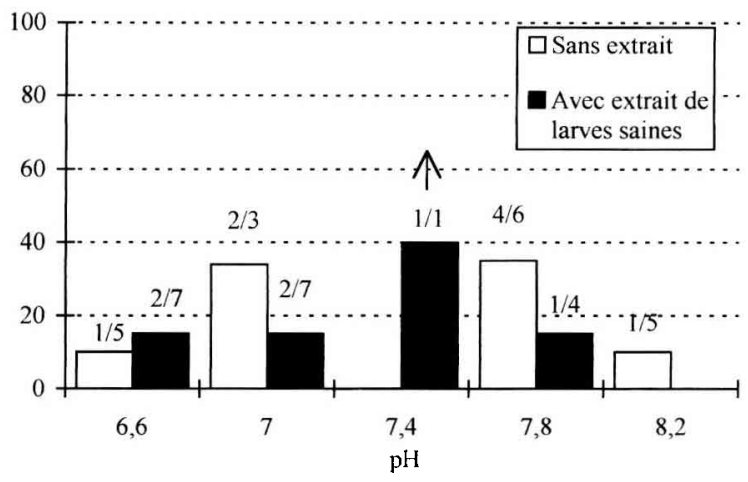

Fig 5. Étude de la survie 18 heures à $23^{\circ} \mathrm{C}$ des phytoplasmes de la FD en fonction $\mathrm{du} \mathrm{pH}$, en présence et en absence d'extrait de larves $E$ variegatus sains. $(\uparrow$ : résultat dépassant la limite supérieure de discrimination de l'épreuve. le rapport au-dessus de chaque barre correspond au nombre de fèves malades sur le nombre total de tèves inoculées par les cicadelles injectées).

$\mathrm{mg} / \mathrm{mL}$ dilué $1 / 2$ ) alors que les meilleurs résultats de survie de phytoplasmes de la FD en absence d'extrait se situent entre $\mathrm{pH} 7$ et 7,8 .

\section{Extraits de plantes}

\section{Extraction des phytoplasmes à partir de fèves malades}

Dans la figure 6 nous donnons à titre de comparaison des résultats d'extraits de phytoplasmes obtenus à partir du sommet d'une fève présentant les symptômes de la maladie : feuille et tige. Les extraits testés en injection au vecteur mettent en évidence la présence de phytoplasmes dans les deux organes. Un meilleur rendement est obtenu avec la tige. Ces résultats confirment les essais antérieurs (Caudwell et al, 1988).

La figure 7 révèle un effet toxique de la tige de fève dans trois essais différents. D'une part, on remarque une nette baisse de la survie des phytoplasmes dans l'essai $V$ qui compare l'extraction avec et sans survie. D'autre part, dans les essais VI et VII, les résultats avec dilutions dans le milieu 1379 en absence d'extrait de fève sont nettement supérieurs aux non dilués.

\section{Effets des différents extraits de plantes saines sur la viabilité des phytoplasmes}

Dans la figure 8 nous avons comparé, pour des survies de 18 heures à $+4{ }^{\circ} \mathrm{C}$, les effets de trois dilutions de chaque extrait végétal $\left(1,1 / 10^{e}\right.$ et $\left.1 / 100^{e}\right)$ à un essai de survie en absence d'extrait végétal. Un effet toxique est évident pour les plus fortes concentrations en extrait végétal, avec la vigne et en particulier avec la fève. Les dilutions des extraits sains améliorent dans tous les cas la survie du phytoplasme. L'extrait de pervenche est le moins toxique, à la dilution $1 / 10^{\mathrm{e}}$ le résultat obtenu est le même qu'en absence d'extrait.

Les résultats de ces expériences indiquent que, dans nos conditions, des différences assez importantes existent entre les extraits sains de ces trois espèces végétales. À poids égal de tissus frais, on peut considerer que la pervenche est la moins toxique en survie de 18 heures à $+4{ }^{\circ} \mathrm{C}$.

\section{DISCUSSION ET CONCLUSION}

Dans ce travail, nous avons recherché et étudié la meilleure aptitude à la survie du phytoplasme de la FD dans les extraits des divers hôtes, animaux ou végétaux, dont il peut être issu. Ces extraits d'hôtes très différents forment des milieux complexes (milieu chimique, milieu physique). Rien n'est connu à propos de ces extraits.

Les résultats concernant les extraits faits à partir de l'hôte animal infectieux (fig 3a), mettent en évidence que (pour un même nombre de

Unité infectieuse x 100

par ml

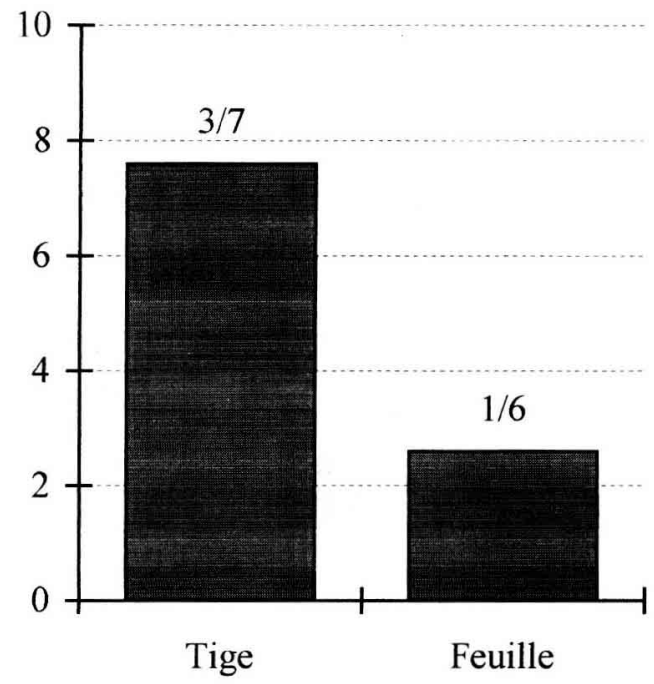

Fève malade

Fig 6. Résultats d'extractions de phytoplasmes de la flavescence dorée de la vigne à partir d'une tige et d'une feuille du sommet d'une fève infectée (Le rapport au dessus de chaque barre correspond au nombre de fèves malades sur le nombre total de fèves inoculées par les cicadelles injectées). 
cicadelles : 6 par $\mathrm{mL}$ ) les mâles sont une meilleure source de phytoplasmes. De même, la survie d'une suspension de phytoplasmes de la FD (fig 3b) dans de l'extrait sain mâle (correspondant à six cicadelles par $\mathrm{ml}$ ) est supérieure à la survie dans l'extrait sain femelle. Ceci a un intérêt pratique : prendre des mâles infectieux comme source de phytoplasmes. Cependant, en égalisant les poids (fig 4) et pour 16,5 mg par $\mathrm{mL}$ (correspondant à la dilution 1/2), l'extrait sain de femelles donne un meilleur résultat que l'extrait sain mâle (B). Ainsi, la dilution de l'extrait sain femelle améliore la survie du phytoplasme. Cette différence de toxicité des extraits sains pourrait être en relation avec la présence des œufs dans

Unité infectieuse $\times 1000$

par $\mathrm{ml}$

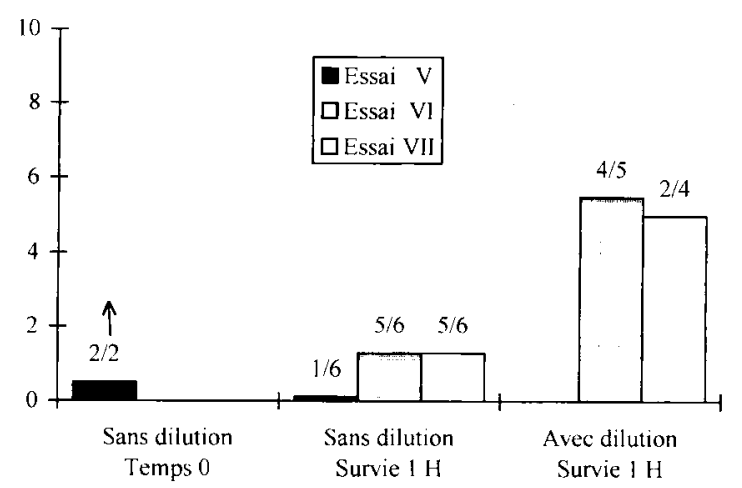

Fig 7. Comparaison de la viabilité du phytoplasme de la FD en présence d'extrait de fèves malades dont il est issu $(\uparrow$ : résultat dépassant la limite supérieure de discrimination de l'épreuve. Le rapport au-dessus de chaque barre correspond au nombre de fèves malades sur le nombre total de fèves inoculées par les cicadelles injectées).

Unité infectieuse $\mathrm{x} 1000$

par ml

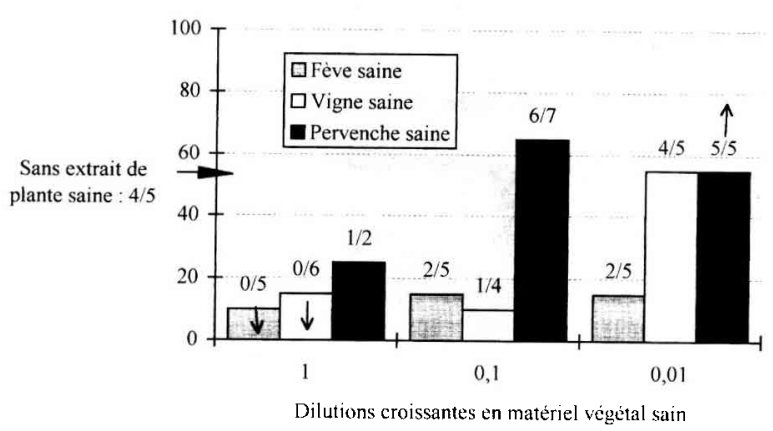

Fig 8. Étude de la toxicité de trois hôtes végétaux sains (fève, pervenche de Madagascar et vigne) sur la survie in vitro pendant 18 heures à $+4^{\circ} \mathrm{C}$, des phytoplasmes de la flavescence dorée de la vigne. ( $\uparrow$ : résultat supérieur et $\downarrow$ : résultat inférieur à la limite de discrimination de l'épreuve. Le rapport audessus de chaque barre correspond au nombre de fèves malades sur le nombre total de fèves inoculées par les cicadelles injectées). les femelles. En effet, pour un même nombre de cicadelles, le poids des femelles fluctue en fonction de la quantité d'œufs. Les œufs n'ont pas de phytoplasmes puisque ceux-ci ne sont pas transmis par les œufs (Caudwell et Larrue, 1977). Ceci suggère que les œufs peuvent être toxiques pour les phytoplasmes de la FD. Pour compléter ces informations, nous avons voulu savoir si les extraits de larves saines pouvaient favoriser la survie du phytoplasme (figs 4 et 5 ). Le meilleur résultat a été obtenu avec l'extrait sain de larves dilué au 1/2. D'autre part, l'étude de l'effet d'un extrait de lanves saines, à différents $\mathrm{pH}$, montre qu'il n'améliore pas la survie du phytoplasme. En absence d'extrait de larves, le $\mathrm{pH}$ optimum pour la survie du phytoplasme de la FD dans le milieu 1379, se situe entre $\mathrm{pH} 7$ et 7,8 .

En ce qui concerne les extraits de phytoplasmes à partir de fève malade, nous avons confirmé que les tiges étaient une meilleure source de phytoplasmes que les feuilles (fig 6). Nous avons donc adopté les tiges pour la suite de ce travail. Puis nous avons constaté (fig 7) que la survie du phytoplasme était bien supérieure lorsque l'extrait infectieux était rapidement dilué dans du milieu 1379 pur. II semblerait que le phytoplasme échappe ainsi à une certaine toxicité des extraits de tissus de la plante hôte.

II nous a paru intéressant de vérifier si la dilution, non plus dans le milieu 1379 pur, mais dans des extraits de l'hôte végétal sain avait le même avantage (fig 8). II résulte de notre travail que les dilutions dans des extraits végétaux ont toujours un effet toxique. Les meilleurs résultats de survie du phytoplasme sont obtenus en diluant la suspension de phytoplasmes dans le milieu 1379 pur, ou dans l'extrait de pervenche saine. Enfin, les extraits sains de fève et de vigne apparaissent toxiques. Nous retrouvons ici un avantage de la pervenche de Madagascar comme hôte de choix pour conserver des souches de phytoplasmes acquises en laboratoire (après inoculation par hétérogreffe ou par cuscute : Cousin et al, 1986 ; Dafalla et Cousin, 1988). La faible toxicité de l'extrait de pervenche, constatée dans le présent travail, pourrait être en relation avec sa sensibilité à de nombreuses maladies à phytoplasmes. Cependant, l'infection de cet hôte par l'insecte vecteur apparaît difficile et nécessite une longue incubation. C'est pourquoi nous avons préféré la fève, qui pousse plus vite, réagit aux piqûres d'une seule cicadelle infectieuse (Caudwell, 1977), et présente des symptômes beaucoup plus rapidement. Dans ce travail, la toxicité de l'extrait de 
fève sur l'infectivité du phytoplasme se révèle être importante d'où la nécessité d'une dilution rapide. Les études menées en Elisa (enzymelinked immunosorbent assay) (Kuszala et al, 1990) n'ont pas mis en évidence d'effet inhibiteur de l'extrait de fève sur l'extraction des antigènes du phytoplasme ni sur leurs réactivités sérologiques. Des antigènes du phytoplasme ont pu être détectés dans toutes les parties de la plante (feuille, tige, racine, radicelle), ainsi qu'une évolution de ceux-ci en fonction de l'âge de l'infection. Concernant la vigne, nous retrouvons ici le problème ancien de l'extraction des agents pathogènes (virus, phytoplasmes) à partir de cette plante, riche en tanins et en acidité. Ceci semble en contradiction avec les symptômes importants que présente cette espèce. Les études menées en Elisa (Kuszala, 1996) sur les trois espèces végétales étudiées, montrent que la vigne se distingue de la pervenche et de la fève, d'une part, par une faible concentration en phytoplasme, et d'autre part, par la nécessité de la présence d'un détergent (Triton $\times 100$, Chaps ou Tween 40) dans le tampon d'extraction. En biologie, ces détergents sont utilisés pour dissocier les protéines de la bicouche phospholipide de la membrane cellulaire. À ce jour, le rôle de ces détergents, dans les extraits bruts de vigne, n'est pas connu. Malheureusement ces conditions sont dénaturantes pour l'intégrité structurale du phytoplasme. En conclusion, il est probable que, pour des raisons de compartimentage, ce qui se passe avec des extraits peut être indépendant de ce qui se passe in vivo. In vivo, le phytoplasme de la FD envahit plus ou moins rapidement, en fonction de sa capacité à annihiler les défenses de l'hôte, les tubes criblés des trois espèces végétales étudiées. La coexistence in vitro lors du broyage de substances compartimentées de façon rigide in vivo entraîne de nombreuses interactions entre les phytoplasmes et les autres constituants. Ainsi, la toxicité des extraits affectant l'infectivité du phytoplasme pourrait être due à l'apparition d'un environnement agressif (physico-chimique) consécutif au broyage et son intensité pourrait être fonction de l'espèce végétale.

Ce travail a montré, pour la première fois, l'interférence des extraits d'hôtes (insectes et végétaux) su. a survie ex vivo d'un phytoplasme. Bien que des investigations supplémentaires soient nécessaires, nous avons pu trouver des conditions expérimentales permettant la meilleure survie du phytoplasme de la FD, selon l'hôte et le $\mathrm{pH}$.

\section{REMERCIEMENTS}

Nous remercions M Caudwell (Inra-Dijon) pour ses critiques et suggestions.

\section{RÉFÉRENCES}

Alivizatos AS, Markham PG (1986) Multiplication of corn stunt spiroplasma in Dalbulus maidis and transmission in vitro, following injection. Ann Appl Biol 108, 545-554

Black LM (1939) Inhibition of virus activity by insect juices. Phytopathology 29, 321-327

Caudwell A (1977) Aspects statistiques des épreuves d'infectivité chez les jaunisses (Yellow's) des plantes et chez les viroses transmises selon le mode persistant. Intérêt de la fève (Vicia faba) comme plante-test pour les jaunisses. Ann Phytopathol 9, 141-159

Caudwell A, Larrue J (1977) La production de cicadelles saines et infectieuses pour les épreuves d'infectivité chez les jaunisses à mollicutes des végétaux. L'élevage d'Euscelidius variegatus KBM et la ponte sur mousse de polyuréthane. Ann Zool Ecol Anim 9, 443-456

Caudwell A, Kuszala C (1986) Mise au point par l'épreuve d'infectivité d'un milieu de survie, d'une méthode de purification et de conservation au froid de l'agent pathogène (MLO) de la flavescence dorée. agronomie 6, 885-892

Caudwell A, Kuszala C, Bachelier JC, Larrue J (1970) Transmission de la flavescence dorée de la vigne aux plantes herbacées par l'allongement du temps d'utilisation de la cicadelle Scaphoideus littoralis. Ball et l'étude de sa survie sur un grand nombre d'espèces végétales. Ann Phytopathol 2, 415-428

Caudwell A, Giannotti J, Kuszala C, Larrue J (1971) Étude du rôle de particules de type «mycoplasme» dans l'étiologie de la flavescence dorée de la vigne. Examen cytologique des plantes malades et des cicadelles infectieuses. Ann Phytopathol 3, 109-125

Caudwell A, Kuszala C, Larrue J, Bachelier JC (1972) Transmission de la flavescence dorée de la fève à la fève par des cicadelles des genres Euscelis et Euscelidius. Intervention possible de ces insectes dans l'épidémiologie du Bois noir en Bourgogne. Ann Phytopathol 181, 189

Caudwell A, Kuszala C, Larrue J (1973) Techniques utilisables pour l'étude de la flavescence dorée de la vigne, Congrès ICVG, Salice Terme Sept, Riv Patol Veg Serg IV, vol IX, 3, 269-276

Caudwell A, Kuszala C, Fleury A (1988) Préparation des antigènes des mycoplasmes (MLO) pathogènes de la flavescence dorée, à partir de tissus végétaux. J Phytopathol 123, 124-132

Cousin MT (1995) Phytoplasmes et phytoplasmoses. agronomie 15, 245-264 
Cousin MT, Sharma AK, Rousseau J, Poitevin JP, Savoure A (1986) Hydrangea virescence. I. Description of the disease and its transmission to the differential host plant Catharanthus roseus by Cuscuta subinclusa. agronomie 6, 249-254

Dafalla GA, Cousin MT (1988) Phyllody of faba bean in the Sudan. I. Progress of symptom expression, host range and transmission to Catharanthus roseus through dodder. agronomie 8, 441-449

Eden-Green SJ (1978) Isolation of acholeplasmas from coconut palms affected by lethal yellowing disease in Jamaica. Zbl Bakt Hyg I Abt Orig A (abstr)

Granata G, Grimaldi V (1991) Electron microscopic detection of mycoplasma-like organisms in epidemic yellow affected grapevines. Petria 1, 171-175

Hamilton MA (1934) Further experiments on the artificial feeding of Myzus persicae Sulz. Ann Appl Biol 22, 243-258

Ivanov PI, Vankova AA (1992) Nutrient medium on the plant extract base for the isolation and cultivation of mycoplasmas. 9th Intern Congr IOM Lett 2, Ames, IA, États-Unis

Kuszala C (1986) Influence du sexe et de l'âge des insectes vecteurs injectés dans l'épreuve d'infectivité des jaunisses des plantes. Mesure radiographique du volume injecté à Euscelidius variegatus (Kirschbaum). agronomie 6, 591-598

Kuszala C (1996) Influence du milieu d'extraction sur la détection du bois noir et de la flavescence dorée de la vigne, par des anticorps poly et monoclonaux dirigés contre les phytoplasmes du stolbur et de la flavescence dorée. agronomie 16, 355-365

Kuszala C, Meignoz R, Caudwell A (1990) Evolution of MLO antigens of grapevine Flavescence dorée according to the age of infection in broadbean, Vicia faba L. Proc 10th Meeting of ICVG Volos, Greece, 3-7 September, 219-224

Lefol C, Lherminier J, Boudon-Padieu E, Larrue J, Louis C, Caudwell A (1994) Propagation of Flavescence dorée MLO (mycoplasma-like organism) in the leafhopper vector Euscelidius variegatus Kbm. J Invertebr Pathol 63, 285-293

Lherminier J, Boudon-Padieu E, Caudwell A (1990) Immunochemistry, a tool for MLO detection in the vector. 8th Intern Congr IOM Lett, Turquie, 1, 219220

Majidi HE, Nazery S (1992) Antibacterial activity of some plant tissue extracts. 8th Inter Conf Plant Path Bact June 9-12, Versailles, france

Meignoz R, Boudon-Padieu E, Larrue J, Caudwell A (1992) Flavescence dorée de la vigne. Présence de MLO et effets cytopathogènes associés, dans le liber de la vigne. J Phytopathol 134, 1-9

Moreau JP (1990) Parasites of plants and insects, the mollicutes transmitted by leafhoppers. Microbiol in Poecilotherms (Biomedical Division)

Sears BB, Schewe G (1994) Optimization of growth conditions for Acholeplasma strain $\mathrm{J} 233$ as a strategy for culturing phytoplasmas in vitro. 10th Intern Congr IOM Lett, France 3, 291-292

Seemüller E, Schneider B, Maurer et al (1994) Phylogenetic classification of plant-pathogenic mycoplasmas by sequence analysis of $16 \mathrm{~S}$ rDNA. 10th Intern Congr IOM Lett, France 224-225

Teranaka M, Okuda S, Suzuki I, Wakaida M (1976) A trial to culture causal agent of plant mycoplasma disease and effect of plant macerating juice on growth of bovine mycoplasma (Mycoplasma sp) and Acholeplasma laidlawii. Bull Coll Agric Utsonomi Univ 1-9 